\title{
Infrared guidance systems. A review of two man-portable defense applications.
}

\author{
Alvaro Pastor \\ Universitat Oberta de Catalunya, Barcelona, Spain \\ alvaropastor@uoc.edu
}

\begin{abstract}
Since the 1950's infrared (IR) guidance technologies have been increasingly used in various fields including scientific, industrial and military applications. Affording visibility despite light conditions, in military applications IR made possible night vision, as well as seeker systems intended to guide missiles autonomously to their targets. This article presents a historical overview of IR guidance and discusses to of its current applications in portable air defense systems.
\end{abstract}

Keywords: Infrared $\cdot$ Guidance $\cdot$ Missile $\cdot$ Homing

\section{Introduction}

Throughout the history of warfare, human senses and reasoning have been the predominant tools used to guide throwing weapons and direct strikes against targets. However, in the mechanized and electronic eras of warfare, new demands emerged regarding quantity of threats, and required speed of response, therefore assistance to human initiative became paramount.

Following the discovery and study of the photoelectric phenomenon during the latter half of the 19th century, scientific efforts in Europe early in the 20th century successfully developed the first elements of infrared (IR) detection for anti aircraft missiles and heat bearing devices. In 1933, E. W. Kutzscher at the University of Berlin, discovered that lead sulphide $(\mathrm{PbS})$ was photoconductive material [1].The period between World Wars I and II is marked by the development of photon detectors and image converters. The image converter, which allowed night vision, was developed on the eve of World War II and became of tremendous interest to the military. Developments were ready for industrial production in 1943 and $\mathrm{PbS}$ was the first practical infrared detector deployed in a variety of applications during the war [2]. These works, that were carried out in secrecy, lead to the fabrication of the most sensitive German IR detectors and its results were only known after 1945. R. J. Cashman led similar efforts in the USA, producing PbS detectors at Northwestern University in $1944[3,4]$.

The IR radiation sources of interest to this research are target aircraft that have to be distinguished from background sources such as the sun, sky and solar reflectance from clouds. On an aircraft the engine produces a large amount of radiant energy which infrared systems are able to detect. The parts of the jet 
aircraft with the largest infrared signatures are the tailpipe and exhaust plume with peak temperatures in the near and middle infrared wave bands. Another source of radiation from an aircraft can be solar reflection from the canopy and other metal parts and in the case of fast jets, aerodynamic heating also plays an important role.

The military IR technology was in its infancy at the end of World War II. After 1945 the German direction of IR research was continued in the USA, Britain and Soviet Union under military sponsorship.After World War II Cashman found that other semiconductors of the lead salt family (PbSe and $\mathrm{PbTe}$ ) showed promise as IR detectors. Immediately after the war, communications, fire control and search systems began to stimulate a strong development effort of lead salt detector technology that has extended to the present day.

An early example of IR systems based on uncooled $\mathrm{PbS}$ detectors applied to air to air missiles was the AIM 9 Sidewinder developed by the USA Navy in mid 50's. On 11 September 1953 the experimental Philco/General Electric XAAM-N7 Sidewinder heat-seeking air-to-air missile scored its first hit. The Sidewinder was later redesignated AIM-9. It entered service in 1956 as the AIM-9B and has been a primary fighter weapon for 60 years. Similarly, Soviet Air Force introduced its Kaliningrad K-5 into service in 1957, an early air to air missile with passive IR homing based on PbS detectors. An improved variant was the Vympel K-13 infrared homing air-to-air missile developed in 1960. Sidewinder and $\mathrm{K}-13$ variants and upgrades remained in active service with many air forces for several decades. From the late 60's and on, with the arrival of the AIM 9D Sidewinder and the Vympel K13M, PbS IR detectors were cooled, which reduced the self generated noise in the detector material.

Discovery of variable band gap HgCdTe ternary alloy by Lawson and colleagues in 1959 opened a new area in IR detector technology as allowed the spectral response of the detector to be custom tailored for specific applications, providing an unprecedented degree of freedom. The bandgap energy tunability results in IR detector applications that span the short wavelength IR (SWIR) 1-3 micrometre, middle wavelength (MWIR) 3-5 micrometre, long wavelength (LWIR) 8-14 micrometre, and very long wavelength (VLWIR) 14-30 micrometre ranges.

Based on the same general principles as the original Sidewinder, in 1955 USA began studies on a small man-portable surface to air missile systems that would emerge as the FIM-43 Redeye MANPADS. The basic Redeye mounted a heat seeker at its homing head that was a direct descendant of the AIM-9B Sidewinder missile, using the same uncooled PbS IR detector unit behind an optical lens [5].

The Soviets started development of two almost identical weapons in 1964, Strela-1 and Strela-2. The man portable 9K32 Strela-2 also known as SA-7b entered service in 1968 and is considered one of the most successful of the portable variants of these early designs.

A major upgrade program for the Redeye started in 1967, as the Redeye II. Testing did not begin until 1975 and the first deliveries of the now renamed FIM-92 Stinger began in 1978. The Soviets likewise improved their own versions, 
introducing the 9K34 Strela 3 in 1974, and the greatly improved dual band IR detectors of 9K38 Igla in 1983 and Igla-S in 2004. In the mid seventies attention turned to the photodiodes for passive IR imaging applications. The main limitation of photoconductive detectors is that they cannot easily be multiplexed on the focal plane. In contrast, photodiodes' easy multiplexing on focal plane silicon chip, allows to be assembled in two dimensional arrays containing more than megapixel elements [6]. After the invention of charge coupled devices (CCDs) by Boyle and Smith [7] the idea of an all solid state electronically scanned two dimensional IR detector array caused attention to be turned to $\mathrm{HgCdTe}$ photodiodes. In the last four decades different types of detectors are combined with electronic readouts to make detector focal plane arrays (FPA). Development in FPA technology has revolutionized infrared imaging. Progress in integrated circuit design and fabrication techniques has resulted in continued rapid growth in the size and performance of these solid state arrays [8].

These systems provide enhanced capabilities like larger number of pixels, higher frame rates, better thermal resolution as well as multiband functionality and other on-chip functions. Systems that gather data in separate IR spectral bands can discriminate both absolute temperature and unique signatures of objects in the scene. By providing this new dimension of contrast, multiband detection also offers advanced band processing algorithms to further improve sensitivity compared to that of single band devices [9].

Due to its remarkable effectiveness in the battlefield it could be argued that passive IR homing represented one of the most significant leaps in tactical technologies of the 20th century.

IR technologies are nowadays widely used in engineering and industrial applications, astronomic observation, medical applications such as thermography or tumor detection, and military applications like night vision, reconnaissance, surveillance, and missile guidance.

\section{MANPADS}

Man-Portable Air-Defence systems (MANPADS) are shoulder-launched surface to air missiles.

MANPADS consist of three major separate elements: The missile in a launch tube which includes the seeker head, an on board infrared seeker; a detachable triggering unit called gripstock; and a unit to supply power and cooling for the missile called the battery coolant unit (BCU). Generally the launch tube includes the sight assembly for acquiring a target, sockets for gripstock and BCU. But in some cases, for example US Stinger, the BCU is inserted into the gripstock, not the launch tube $[9,10]$.

In order to engage a target, an operator of any popular passive homing MANPADS must first insert the BCU to power the seeker head. Then use the sights on the launch tube to aim at a target until its acquisition by the IR detector which alerts the operator using a lock-on tone. Next, the trigger on the gripstock is pulled to the first position which uncages the IR seeker head, allowing 
the seeker to rotate, being capable to aim above and ahead of the target. Some MANPADS automatically calculate where to aim, based on aircraft speed; some have television or infrared scopes to aid in target acquisition; and all need to lock on to the target prior to launch to increase the probability of hitting the aircraft. Finally, the operator must pull the trigger to the second position in order to launch the missile. Once lock-on has been achieved and the missile has been launched, the seeker provides the guidance information to the auto pilot of the missile and there is no further need of an operator. On launch the missile is initially ejected from the tube by a small charge. Once the missile is a safe distance from the operator the boost motor ignites taking the missile to its top speed then a sustain motor to maintain that speed. The warhead of the missile is fully armed at this point. The missile has an auto pilot which relies on stabilising tail fins and two control fins to manoeuvre the missile to the target. There may be an impact fuse on the warhead or a proximity fuse, and in addition, a delayed self destruct mechanism set from the time of missile launch.

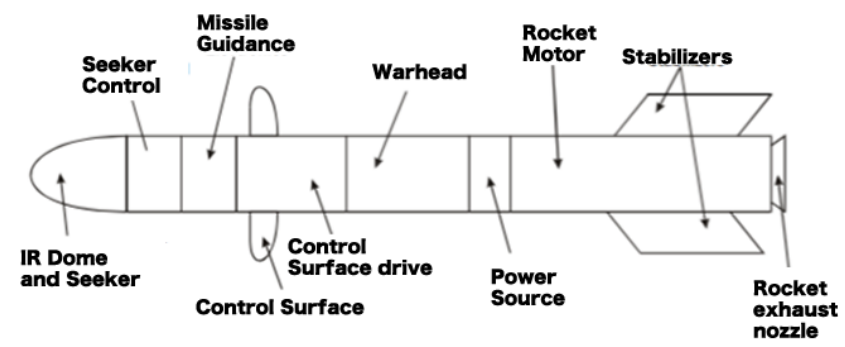

Fig. 1. A typical missile with an IR dome, seeker, guidance and control system, warhead, and rocket motor with rollerons for maneuvering and tail fins for stability.

Among MANPADS main attractions are its compactness, weight, and ease of operation: Most are about 1.5 meters long, weigh between 15 and 20 kilograms fully loaded, and can be quickly assembled and aimed, some requiring less than 30 seconds. The missiles can be effective up to 6 kilometers in range approximately. While the launch tubes are reusable in principle, they are not intended to be reloaded with a missile on the battlefield.

\subsection{Guidance systems}

Nearly all MANPADS are designed using a passive homing architecture, that is that passively tracks the infrared (IR) emissions of an aircraft to then impact its target or deliver its payload in nearby locations. This article focuses the current developments of this paradigm. The guidance section consists of a seeker assembly, a guidance assembly, a control assembly, a missile battery, and four control surfaces that provide in-flight maneuverability. The IR seeker is located at the 
front of the missile detects radiation in ranges from 750 micrometre to 1 milimetre generally focused a different wavelengths depending on the temperature of the source. A key characteristic of passive homing MANPADS is that once fired, they require no more input from any operator, provided its fire-and-forget capability. The US Redeye and Stinger, the French Mistral and the Russian Strela and Igla families of MANPADS rely on passive IR homing. By virtue of reverse engineering, many versions of these systems have been produced, such as Chinese QW-1, Pakistan Anza MKII and the Iraninan Misagh family. In sum it is estimated that nearly 500,000 of these systems have been produced worldwide and are used to present date among state and non state actors [11]. A review of two important MANPADS based on passive IR homing will be provided in the next section.

A less common architecture, command guidance, would rely on outsourcing the tracking of the target to an allied third party, who then communicates guidance commands to the missile and thus directs it to the target. This means that the gunner needed to track both the missile and the target, and had to steer the missile to the target manually. In later members of the series, the missile is tracked automatically by the launching unit, which also assists the gunner in tracking the target. This approach of guidance is called semi-automatic command to line-of-sight (SACLOS). Known MANPADS to rely on this type of command guidance is the Swedish RBS-70 family and the British Javelin SAM and Starstreak families which use a laser beam to guide the missile to its target. Generally, command guided missiles are far less common and have been tested much less in action than the passive homing variants [12].

An exception to these main alternatives is the use of semi-active homing system such as Chinese FL-2000B. Semi-active homing, while unusual for MANPADS, is frequently employed in other precision- guided munitions, like laserguided bombs or missiles. It requires that the target is illuminated by an outside source, for instance a ground- based laser. The missile is equipped with a seeker focused on the bandwidth of the reflected laser light.

\subsection{First generation}

IR guided missiles were first developed in the 1960s and used rotating reticles as optical modulators. The purpose of optical modulation is to provide tracking information for the IR seeker. Reticles are also able to suppress background emission with regards to the target by a process known as spatial filtering. The purpose of spatial filtering is to maximise the signal to noise ratio of the target with regard to the background radiation. As the target is a hot point source its signal will be a series of pulses with a chopping frequency which depends on the arrangement of opaque and transparent spokes of the reticle and its rotational frequency. The background will cover many spokes so it will signal an extended source with no chopping. The signal is then amplified and electrically filtered with a band-pass filter centred at the chopping frequency suppressing the

background radiation. This produces an error signal giving guidance information in the form of polar coordinates projected onto the image plane. 
The simplest IR seekers and the most widely proliferated have rotating reticles. They are known as spinscan seekers and use uncooled PbS detectors that work in the 2-2.7 micrometre waveband. This means they can only detect the high temperature tailpipe and exhaust plume essentially limiting them to tail chasers. The first generation of IR seeker heads had a rotating rectangular field of view (FOV) with a single detector element, leading to increasing inaccuracy in close proximity to the target. The dome of the seeker requires to be made of an IR transmitting material, generally silicon, germanium or a variant of zinc seleneide as Irtran. Once the IR radiation passes through the dome a Catadioptric telescope reflects the signal onto a rotating reticle.

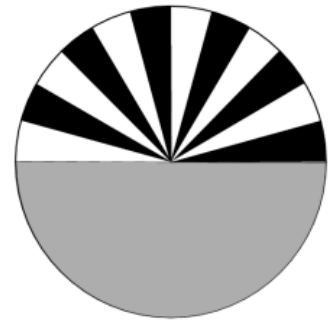

a

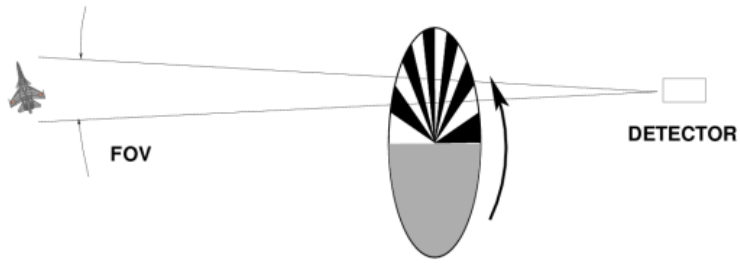

b

Fig. 2. (a) An early rotating reticle for background suppression and directional information known as rising sun pattern. (b) Consider the reticle to be rotating at a constant rate. Its action will result in different detector outputs for the point target and for the background. The resultant ouput signal is a sine wave in which the amplitude corresponds to the magnitude of tracking error, and the phase of the signal corresponds to the direction of the tracking error. The pulses corresponding to the target may then be easily separated by electronic filtering using a narrow bandpass filter at the pulse frequency, thus enabling the required discrimination between the target and background.

A well known example of these systems is the Strela-2 also known as SA-7b which has proliferated worldwide and seen use in nearly every conflict since it first entered service. Several countries have produced their own variant through reverse engineering. Copies include; the Chinese HN-5, Pakistani Anza MKI and Egyptian Sakr Eye. These variants may have different missile characteristic such as improved signal processing for better tracking.

However, there are several problems associated with this early approach. Because of the detector waveband it tracks the hottest part of the target, usually tailpipes of jet engines. Therefore, even in a successful engagement, the aircraft could still land as the small warhead may fail to damage the engine. Also, it can lose target tracking due to solar reflectance and be easily defeated by expendable off board countermeasures [13]. 
Early models of the Strela or Redeye families, scanned in just one range of the spectrum, initially in the $2-3$ micrometre band [14]. While this enables the seeker to distinguish between the IR radiation of the earth which is approximately 10 micrometre, the sun around 3 micrometre, and a fighter jet located at 2 micrometre for the tailpipe, 4 micrometre for the aft airframe and 4-8 micrometre for the exhaust plume, it can easily be fooled by ares designed to radiate in this spectrum [15]. Spinscan seekers also exhibited on-axis insensitivity, which was overcome by rotating the optics instead of the reticle, a method known as conical scanning.

\subsection{Second generation}

Second generation seekers overcame the problem of on-axis insensitivity by rotating the optics instead of the reticle, which is known as conical scanning (CONSCAN). In this arrangement the secondary mirror is tilted and the reticle is a full wagon wheel which produces a nutation circle and frequency modulation (FM) of the image. The magnitude of the frequency modulation gives the offaxis distance and a pick-off gives phase variation to yield the position of the target in the field of view. Instead of the uncooled $\mathrm{PbS}$ detectors used in earlier models second generation seekers use cooled $\mathrm{PbS}$ or Indium Antimonide (InSb) detectors which allowed them to operate in the 3-5 micrometre range, perceiving cooler parts of the plume and hot components of the aircraft, thus widening the tracking aspect. Newer generation models as Strela 3 switched to the 3-5 micrometre range, in which to cool the detector argon or nitrogen is used [14].
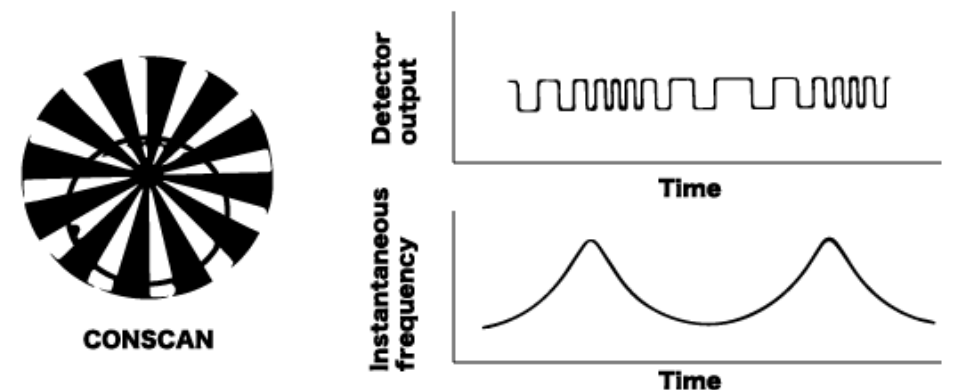

Fig. 3. In CONSCAN seekers, when the target is centered in the seeker, the detector gives a pulsed output similar to the spin-scan. The con-scan seeker provides frequency modulation at small tracking errors and amplitude modulation at large tracking error when the nutation cycle of the target image is off the reticle during a part of scan cycle.

An example of this type of system is the Russian SA-14 which entered in service in 1974 as an improved version of the SA-7b with better tracking and 
a wider aspect capability. It could track cooler targets at greater ranges and engage faster moving targets [10]. Also the SA- 16, brought into service in 1981 was still based on the same CONSCAN IR seeker. Another type of seeker uses four detectors in an open-cross formation instead of a reticle. It still uses a nutation circle to give FM by producing four equally spaced detector outputs for an on-axis target and unequal spacing for off-axis targets. An example of this type of system is the original French Mistral. All the variants of second generation MANPADS have different maximum slant range, altitude and speed. Some may contain a capabilities to counter the countermeasures deployed by the target [16]. Further development of the detectors and electronic components caused the emergence of new seeker types used in the MANPADS.

\subsection{Third generation}

In order to be more resistant to target counter measures new scanning techniques were devised to replace the conventional reticle system. Their aim is spectral sensitivity tailored to closely match the emission signatures of military vehicles, such as aircrafts, and reject other sources. They can discriminate targets from clutter and countermeasures but use digital signal processing to cope with the large amount of data [17].

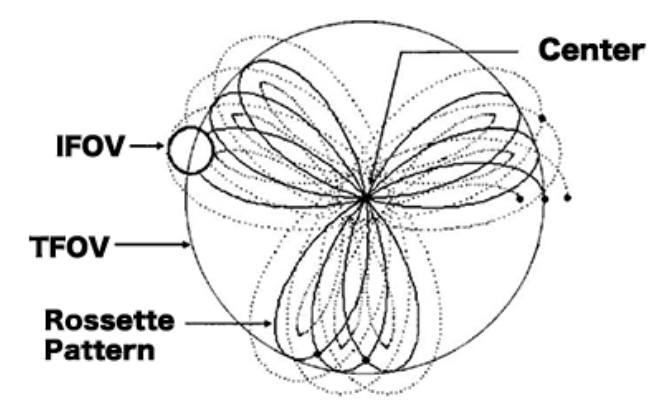

Fig. 4. Semi-imaging seekers, scans the total FOV (TFOV) in a specific pattern with the help of a single detector and an optical scanning system. The detector has a very small instantaneous FOV (IFOV). At the end of the scan period a partial image of the scene is obtained. The radiance values of sampled spatial locations from the scene are processed directly, without forming an actual image, to perform target position estimation. An example of semi-imaging seekers is the rosette scanning seeker.

These new IR seekers are termed pseudo-imaging as they create a simulated image of the target by moving the infrared signal over the detector. Two offset mirrors rotate to produce a specified pattern, the most common one being the rosette scan as this visits the axis multiple times for each rotation. Thus, seeker 
heads used a very narrow FOV that moved in a rosette pattern to improve the information available to the guidance system.

In the single detector rosette scanning seekers, the seeker samples the IR scene with a specific scanning pattern but instead of forming an image with the acquired samples, other signal processing techniques are employed to estimate the targets position [12].

Along with new scanning techniques multispectral detectors were also incorporated. Multispectral IR provides target identification under conditions where one wavelength is unable to identify the target. They could be ultraviolet (UV)/IR or short wave and medium wave IR (SWIR/MWIR). This ability to detect at different wavelengths made the discrimination between target and countermeasure much easier. Also called dual color seekers, this paradigm could be found using either two bands in the IR spectrum or a combination of IR and a band from a completely different spectrum, like ultraviolet (UV) radiation, millimeter waves or visible light. Four colours may be maximum number of bands that can be stacked in a single pixel. These models used InSb or mercury cadmium telluride ( $\mathrm{HgCdTe})$, which required to be cooled to around negative 200 degrees Celsius to achieve sufficient sensitivity, as well as cadmium sulfide (CdS), which covers part of the UV spectrum $[18,19]$. Latest image fusion techniques combine the best of both images such as high resolution of SW with temperature sensitivity of LW.

Examples of third generation MANPADs are the Russian Igla SA-18 and SA24, US Stinger FIM-92B featuring Passive Optical Seeker Technique (POST), and the US Stinger series that features its Reprogrammable Micro Processor (RMP), starting with FIM-92C and later variants. At least two other countries have produced their own variants, the Chinese QW-18 and Iranian Misagh family.

\subsection{Fourth generation}

Latest works on MANPADS lead to the development of fourth generation seekers, or imaging seekers, which produce an image of the target on a focal plane array. This is either done by a linear array which is scanned across the scene or a 2-d staring array. This IR imaging may be understood as closer to images from a digital camera, thus a wide array of computer vision algorithms became available. The computer makes the comparison between the model projected in a $2 \mathrm{D}$ space depending on the aiming conditions given by the measurement unit, with the result of an edge detection algorithm processed on the image seen by the seeker. The image is procesed with automatic detection and target tracking algorithms to increase efficiency of accurate terminal guidance [20]. However in such a design the cost of obtaining the required image size for sufficient resolution and the computing power needed for the image processing in real time is extremely high. As of now these IR detector systems are bulky, heavy and consume a lot of power relative to comparable alternatives. Therefore this type of MANPADS are significantly less common in current battlefields [12]. 


\section{Review of two systems}

While the FIM-92 relies on an infrared homing guidance system like the Redeye before it provides for better tracking and countermeasure avoidance. The Stinger FIM 92B featuring POST began its development in the early 80s and its production was terminated at the end of the decade. Stinger FIM-92C and later variants that feature RMP are characterized by its ease of reconfiguration in order to successfully engage with targets despite to new types of countermeasures being used. Its production began in the late 80 's while it is still currently in service worldwide. In 2019, the U.S. Army began retrofitting its Stinger missiles with proximity fuzes, which allow missiles to destroy unmanned airborne systems with direct hits or by detonating near them.

The 9K338 Igla-S is one the latest variants in the Russian Igla MANPADS family which began its production in the early $80 \mathrm{~s}$. The $\mathrm{S}$ variant began its development in 2004 and then represented important improvements with respect to its predecessors featuring more sensitive seeker techniques and improved resistance against countermeasures.

Both of these systems are based on combined IR/UV seeker that uses FM modulated scanning, achieving higher resistance to environmental disturbances and enemy countermeasures. A summary of their features is presented in 1.

Table 1. Technical features of two of the most popular passive homing MANPADS.

\begin{tabular}{|l|l|l|l|l|c|}
\hline System & Weight $(\mathrm{kg})$ & Range $(\mathrm{km})$ & Altitude (feet) & Seeker type & Scan type \\
\hline Stinger FIM-92 & 15 & $0.2-4.8$ & 10000 & $\begin{array}{c}\text { Pseudo-imaging } \\
\text { Dual-band } \\
\text { Pseudo-imaging } \\
\text { Dual-band }\end{array}$ & $\begin{array}{c}\text { FM } \\
\text { modulated } \\
\text { FM } \\
\text { modulated }\end{array}$ \\
\hline
\end{tabular}

A FIM-92 MANPADS weigh approximately $15 \mathrm{~kg}$ ready for action, has a reported minimum range of $0.2 \mathrm{~km}$ and a maximum range of $4.8 \mathrm{~km}$. The maximum altitude reachable by its missile is reported as 10 thousand feet, at 2.2 mach maximum speed. The seeker type in use is third generation dual band IR and UV, argon-cooled InSb, capable of all round engagement aspect.

A 9K338 Igla S ready for action weighs approximately $17.9 \mathrm{~kg}$. The launcher unit fires the missile $9 \mathrm{M} 342$ capable of an operative minimum range of $0.6 \mathrm{~km}$ and a maximum range of $6 \mathrm{~km}$. Its fuse type allows delayed impact, magnetic and proximity activation. The seeker type in use is third generation dual band $\mathrm{IR}$ and UV, argon-cooled or nitrogen-cooled $\mathrm{PbS} / \mathrm{InSb}$, capable of all round engagement aspect [21].

\section{Conclusion}

The evolution of IR guidance and its current MANPADS applications are the result of an almost 100 years search for autonomous guiding systems, which 
disregarding the conditions of the scene, day or night and all weather, could successfully strike fast targets without compromising human operators. Among fire and forget MANPADS, Igla-S system may be considered substantially more efficient than its Igla predecessors and the US Stinger FIM-92 MANPADS due mainly to its unique operation range of $6 \mathrm{~km}$, its support for both contact and proximity fuzes, and its improved guidance and control algorithms which ensure precise navigation and selection of the most optimal moment for the warhead's explosion for both in the contact and proximity modes.

Emerging requirements include improved ranges in various atmospheric conditions with recognition ranges of more than $20 \mathrm{kms}$ are driving continuous developments in the field. It is foreseeable that next generation IR detectors will have larger numbers of pixels, frame rates of more than $250 \mathrm{~Hz}$, multispectral detection including 3-4 bands with low crosstalk, on-chip processing implementing well testesd computer vision algorithms such as segmentation, edge and motion detection. They may be able to operate near room temperature operation to reduce power consumption by getting rid of the dewar and cooler [22].

To increase reliability and reduce complexity, alternate substrates for affordable larger format IR FPA may be developed. However, HgCdTe is unlikely to be seriously challenged for high performance applications, due that it affords multispectral capabilities and fast response. Only a relatively new alternative IR material system, InAs/GaInSb has great potential for LWIR/VLWIR spectral ranges with performance comparable to $\mathrm{HgCdTe}$. It may be predicted that hot $\mathrm{HgCdTe}$ technology will remain a central paradigm because of its established fabrication processes and customization of range of operation. Since its spectral wavelengths can be tuned, this material will have its presence in almost all the spectral bands from short to very long wavelength bands [23].

Other improvements may occur in the fields of high dynamic range operation and curved FPAs. In addition some developments to take place in digital FPAs, active pixels and foveas/region of interest (ROI), 3D range gated imaging and the application of artificial intelligence are also current topics of research in this field $[24,25]$. The proliferation of integrated, accurate sensors provides the opportunity to create a detailed, cohesive picture of the battlefield that could enhance and accelerate combat decisions.

\section{References}

1. A. Rogalski. History of infrared detectors. Opto-Electronics Review, 20:279-308, 2012.

2. Jianhua Lu and Ruifeng Wang. The implementation method and the development tendency of infrared stealth technology. In Applied Optics and Photonics China, 2015.

3. M.Krake and H. Rothe. German infrared and night vision technology: from the beginning until 1945. Proc. SPIE 7780, Detectors and Imaging Devices: Infrared, Focal Plane, Single Photon, 2010.

4. E. W. Kutzscher and E. S. Brumfield. Compact infrared search system. In United States Patent 3219824, 1965. 
5. M. Cagle. History of the redeye weapon system. Historical Division US Army Missile Command, 1974.

6. Doğan Uğur Sakarya. Optical design of dual-mode seeker for long-wave infrared and mid-wave infrared seeker in missile application. In Optical Engineering + Applications, 2018.

7. W. S. Boyle and G. E. Smith. Charge coupled semiconductor devices. Bell Syst. Tech. J., 49:587-593, 1970.

8. M. A. Kinch. Fifty years of hgcdte at texas instruments and beyond. In Defense + Commercial Sensing, 2009.

9. L.P. Lazarev. Infrared and Light Wave Aircraft Homing and Guidance Apparatus. Defense Technical Information Center, 1968.

10. M. S. Ab-Rahman and M. Hassan. Lock-on range of infrared heat seeker missile. 2009 International Conference on Electrical Engineering and Informatics, 02:472477, 2009.

11. D.J. van Ovost. Global Mobility: Anywhere, Anytime, Any Threat? Countering the MANPADS Challenge. USAF Center for Strategy and Technology, 2012.

12. S. Campana, J. S. Accetta, and David L. Shumaker. Passive ellectro-optical systems. In The Infrared \& Electro-Optical Systems Handbook Volume 5, 1993.

13. J. Milthorpe and P. Lynn. Effect of aerodynamic heating on infrared guided missiles. School of Aerospace and Mechanical Engineering,, 2005.

14. M. Fiszer and J. Gruszczynski. On arrows and needles. Journal of Electronic Defense, page 46, 2002.

15. C. Kopp. Heat-seeking missile guidance. Australian Aviation, 1982.

16. C. Willers and J. P. Delport. Csir optronic scene simulator finds real application in self-protection mechanisms of the south african air force. In CSIR 3rd Biennial Conference, 2010.

17. W. M. Caplan. Ir imaging seekers may be very resistant to laser jamming. Spie Newsroom, 2014.

18. J. W. Lyons, D. Long, and R. Chait. Critical Technology Events in the Development of the Stinger and Javelin Missile Systems: Project Hindsight Revisited. National Defense University Press, 2012.

19. H. Uzeler, S. Çakir, and T. Aytaç. Image generation for single detector infrared seekers via compressive sensing. In Proceedings of SPIE, 2013.

20. Y. Long-po. Analyses on infrared optoelectronics recognition technology in missile defense system. In Department of Early Warning $\&$ Surveillance Intelligence. Air Force Radar Academy, 2009.

21. C. Bates, J. Lucas, and J. Robinson. The javelin integrated flight simulation. In International Conference on Computational Science, 2001.

22. Y. Reibel, L. Espuno, R. Taalat, A. Sultan, P. Cassaigne, and N. Matallah. High performance infrared fast cooled detectors for missile applications. In SPIE Defense + Security, 2016.

23. H. Dai, Y. Xu, and S. L. Zhao. Infrared recognition technology and its development trend in the middle segment of ballistic missile. In 4th International Conference on Machinery, Materials and Information Technology Applications, 2016.

24. W. Qiu, K. Wang, S. Li, and K. Zhang. Yolo-based detection technology for aerial infrared targets. 2019 IEEE 9th Annual International Conference on $C Y$ BER Technology in Automation, Control, and Intelligent Systems (CYBER), pages 1115-1119, 2019.

25. B. Zhao, S. Xu, J. Guo, Ruimin Jiang, and J. Zhou. Integrated strapdown missile guidance and control based on neural network disturbance observer. Aerospace Science and Technology, 84:170-181, 2019. 